\title{
TORSION-FREE SUBGROUPS OF TRIANGLE GROUPS
}

\author{
R. D. FEUER
}

\begin{abstract}
Certain torsion-free subgroups of various triangle groups are considered, the proof of their existence, and in some cases their calculation outlined. There is one section which treats certain specific triangle groups, and one which treats the general case.
\end{abstract}

1. Introduction and summary. W. Fenchel posed the problem to show that every finitely generated Fuchsian group has a torsion-free subgroup of finite index. (If this is true, the group also has a normal torsion-free subgroup of finite index.) Fenchel's problem was solved in full first by R. Fox [5] and later, in a simplified form, by J. Mennicke [6] who reduced the general problem to that of the "triangle groups" $(r, s, t)$ defined as

$$
(r, s, t)=g p\left\langle a, b, c ; a^{r}=b^{s}=c^{t}=a b c^{-1}=1\right\rangle .
$$

Both Fox and Mennicke use the geometrically obvious fact that in a Fuchsian group, in the standard presentation given by $\mathrm{R}$. Fricke [8], the elements of finite order are conjugates of the generators of finite order and their powers. A group theoretical proof, excluding only a finite number of cases, was given recently by J. McCool [7] and by P. Schupp (still unpublished). To solve Fenchel's problem it then suffices to show that there exists a finite homomorphic image of the given Fuchsian group in which the maps of the generators have the same orders as those indicated by the relations defining the Fuchsian group. For the groups $(r, s, t)$ we give a new and very simple construction of such a finite group in \$3. However, the order of this finite group (which equals the index of a normal torsionfree subgroup in the triangle group) is very large, and we show in $\$ 2$ that, at least in some cases, we can find nonnormal torsion-free subgroups of $(r, s, t)$ which are of much smaller index and which are, in a certain sense, maximal. Using the approach established by $A$. Hurwitz [3] in his investigation of Riemann surfaces we may describe these results as follows: We construct Riemann surfaces of minimal genuses which have branch points over exactly 3 points in

Received by the editors August 25, 1970.

AMS 1970 subject classifications. Primary 20H10; Secondary 20H10, $20 \mathrm{~B} 05$.

Key words and phrases. Reidemeister-Schreier method, discontinuous groups, noneuclidean geometry, Riemann surfaces.

Copyright (c) 1971, American Mathematical Society 
the complex plane and which have the property that the conformal map of the canonically dissected surface in the noneuclidean plane can be tesellated by replicas of the fundamental region of the group $(r, s, t)$.

2. Torsion-free subgroups of small index in triangle groups. Three cases are considered. They are:

(1) $t=2, s=3, p$ an arbitrary prime $\geqq 7$.

(2) $t=3, s=5, p$ an arbitrary prime $\geqq 7$.

(3) $t=2, s=5, p$ an arbitrary prime $\geqq 7$.

TABLE of Permutation $\bar{b}$

\begin{tabular}{|c|c|c|}
\hline $\begin{array}{c}\text { Triangle } \\
\text { Group }\end{array}$ & Value of $p$ & Permutation $\bar{b}$ \\
\hline \multirow{2}{*}{$(2,3, p)$} & $p \equiv 1 \bmod 6$ & $\begin{aligned} \bar{b}=\prod_{m=0}^{(p-1) / 6-1}[(3 m+1, p-(3 m+1), p-(3 m+2)) \\
\cdot(3 m+3,3 m+2, p-(3 m+3))]\end{aligned}$ \\
\hline & $p \equiv 5 \bmod 6$ & $\begin{aligned} \bar{b}= & \prod_{m=0}^{(p-5) / 6-1}[(3 m+1, p-(3 m+1), p-(3 m+2)) \\
& \cdot(3 m+3,3 m+2, p-(3 m+3))]\left(\frac{p}{2}-\frac{3}{2}, \frac{p}{2}+\frac{3}{2}, \frac{p}{2}+\frac{1}{2}\right)\end{aligned}$ \\
\hline \multirow{4}{*}{$(3,5, p)$} & $p \equiv 1 \bmod 12$ & $\bar{b}=\prod_{m=0}^{(p-13) / 12}(p-(4 m+1), p-(4 m+4), 8 m+6,8 m+3,8 m)$ \\
\hline & $p \equiv 5 \bmod 12$ & $\begin{aligned} \bar{b}= & \prod_{m=0}^{(p-17) / 12}[(p-(4 m+1), p-(4 m+4), 8 m+6,8 m+3,8 m)] \\
& \cdot\left(\frac{2}{3} p+\frac{2}{3}, \frac{2}{3} p-\frac{1}{3}, \frac{2}{3} p-\frac{4}{3}, \frac{2}{3} p-\frac{7}{3}, \frac{2}{3} p-\frac{10}{3}\right)\end{aligned}$ \\
\hline & $p \equiv 7 \bmod 12$ & $\bar{b}=\prod_{m=0}^{(p-19) / 12}[(p-(4 m+1), p-(4 m+4), 8 m+6,8 m+3,8 m)]$ \\
\hline & $p \equiv 11 \bmod 12$ & $\begin{aligned} \bar{b}= & \prod_{m=0}^{(p-23) / 12}[(p-(4 m+1), p-(4 m+4), 8 m+6,8 m+3,8 m)] \\
& \cdot\left(\frac{2}{3} p+\frac{8}{3}, \frac{2}{3} p-\frac{1}{3}, \frac{2}{3} p-\frac{10}{3}, \frac{2}{3} p-\frac{19}{3}, \frac{2}{3} p-\frac{22}{3}\right)\end{aligned}$ \\
\hline
\end{tabular}




\begin{tabular}{|c|c|c|}
\hline $\begin{array}{c}\text { Triangle } \\
\text { Group }\end{array}$ & Value of $p$ & Permutation $\bar{b}$ \\
\hline \multirow{4}{*}{$(2,5, p)$} & $p \equiv 1 \bmod 8$ & $\bar{b}=\prod_{m=0}^{(p-9) / 8}(p-(5 m+1), p-(5 m+3), p-(5 m+5), 3 m+2,3 m)$ \\
\hline & $p \equiv 3 \bmod 8$ & $\begin{aligned} \bar{b}= & \prod_{m=0}^{(p-19) / 8}[(p-(5 m+1), p(5 m+3), p-(5 m+5), 3 m+2,3 m)] \\
& \cdot\left(\frac{3}{8} p+\frac{47}{8}, \frac{3}{8} p-\frac{9}{8}, \frac{3}{8} p-\frac{17}{8}, \frac{3}{8} p-\frac{25}{8}, \frac{3}{8} p-\frac{33}{8}\right) \\
& \cdot\left(\frac{3}{8} p+\frac{39}{8}, \frac{3}{8} p+\frac{31}{8}, \frac{3}{8} p+\frac{23}{8}, \frac{3}{8} p+\frac{7}{8}, \frac{3}{8} p-\frac{1}{8}\right)\end{aligned}$ \\
\hline & $p \equiv 5 \bmod 8$ & $\begin{aligned} \bar{b}= & \prod_{m=0}^{(p-13) / 8}[(p-(5 m+1), p-(5 m+3), p-(5 m+5), 3 m+2,3 m)] \\
& \cdot\left(\frac{3}{8} p+\frac{17}{8}, \frac{3}{8} p+\frac{9}{8}, \frac{3}{8} p+\frac{1}{8}, \frac{3}{8} p-\frac{7}{8}, \frac{3}{8} p-\frac{15}{8}\right)\end{aligned}$ \\
\hline & $p \equiv 7 \bmod 8$ & $\begin{aligned} \bar{b}= & \prod_{m=0}^{(p-15) / 8}[(p-(5 m+1), p-(5 m+3), p-(5 m+5), 3 m+2,3 m)] \\
& \cdot\left(\frac{3}{8} p+\frac{27}{8}, \frac{3}{8} p+\frac{11}{8}, \frac{3}{8} p-\frac{5}{8}, \frac{3}{8} p-\frac{13}{8}, \frac{3}{8} p-\frac{21}{8}\right)\end{aligned}$ \\
\hline
\end{tabular}

We denote the triangle group as $G$. The first step is to calculate a subgroup $H$ in $G$ of index $p$, with the powers of $a$ as coset representatives. This is done by the use of a quotient permutation group $\bar{G}$ of $G$ on the $p$ symbols $0,1, \cdots, p-1$, and by the application of the Reidemeister-Schreier method. In the homomorphism from $G$ to $\bar{G}$, the image $\bar{a}$ of $a$ is always the cycle $(0,1,2, \cdots, p-1)$. The images $b$ of $b$ that I use are listed under the Table of Permutations $b$ (see above).

We consider the subgroup of $\bar{G}$ which leaves the symbol 0 fixed. The preimage of this group in $G$ will be our group $H$. The kernel $N$ of the map from $G$ to $\bar{G}$ is the fundamental group of a closed twodimensional manifold. The groups $H / N$ are listed in the "Summary" below.

It should be noted that all subgroups of $G$ of the same index of $N$ are isomorphic to $N$.

It is obvious that any permutation of the orders of $a, b$, and $a b$ will leave the group unchanged up to isomorphism, and that therefore the sequential order of the numbers in the parentheses is unimportant. 
SUMMARY OF RESULTS

$p$ represents an arbitrary prime $\geqq 7$

\begin{tabular}{|c|c|c|c|}
\hline$G$ & Value of $p$ & Index of $N$ in $G$ & $H / N$ \\
\hline \multirow{2}{*}{$(2,3, p)$} & $p \equiv 1 \bmod 6$ & $12 p$ & dihedral, order 12 \\
\hline & $p \equiv 5 \bmod 6$ & $12 p$ & abelian, 3, 2, 2 \\
\hline \multirow{4}{*}{$(3,5, p)$} & $p \equiv 1 \bmod 12$ & $60 p$ & $A_{5}$ \\
\hline & $p \equiv 5 \bmod 12$ & $15 p$ & cyclic, order 15 \\
\hline & $p \equiv 7 \bmod 12$ & $15 p$ & cyclic, order 15 \\
\hline & $p \equiv 11 \bmod 12$ & $15 p$ & cyclic, order 15 \\
\hline \multirow{4}{*}{$(2,5, p)$} & $p \equiv 1 \bmod 8$ & $60 p$ & $A_{5}$ \\
\hline & $p \equiv 3 \bmod 8$ & $20 p$ & dihedral, order 20 \\
\hline & $p \equiv 5 \bmod 8$ & $20 p$ & dihedral, order 20 \\
\hline & $p \equiv 7 \bmod 8$ & $20 p$ & dihedral, order 20 \\
\hline
\end{tabular}

3. Quotient groups of triangle groups, as subgroups of $\operatorname{SPL}(2, q), q$ an appropriately chosen power of a prime. Let $p$ be a prime, $q$ a power of $p . S P L(2, q)$ denotes the group of substitutions

$$
z^{\prime}=(\alpha z+\beta) /(\gamma z+\delta), \quad \alpha \delta-\beta \gamma=1,
$$

where $\alpha, \beta, \gamma, \delta$ are in the Galois field of order $q$. We prove the following.

Lemma. If there exists a $\rho, \rho \neq 0, \pm 1$, such that $\alpha+\delta=\rho+\rho^{-1}$, then the substitution (1) is of order $n$ if the order of $\rho$ in the multiplicative group of the Galois field is $2 n$, and $p \neq 2$. For $p=2, n$ is odd (as a divisor of $q-1)$ and is equal to the order of $\rho$.

Proof. The fixpoints of (1) are given by

$$
\gamma z^{2}+(\delta-\alpha) z-\beta=0 .
$$

Let $\gamma=0$. Then $\alpha \delta=1, \alpha+\delta=\rho+\rho^{-1}, \quad(\alpha-\delta)^{2}=\left(\rho-\rho^{-1}\right)^{2}$, and $\alpha-\delta= \pm\left(\rho-\rho^{-1}\right)$. Hence either $\alpha=\rho, \delta=\rho^{-1}$ or $\delta=\rho, \alpha=\rho^{-1}$. We have 


$$
\left(\begin{array}{ll}
\rho & \beta \\
0 & \rho^{-1}
\end{array}\right)^{k}=\left(\begin{array}{cc}
\rho^{k} & \frac{\rho^{k}-\rho^{-k}}{\rho-\rho^{-1}} \beta \\
0 & \rho^{-k}
\end{array}\right)
$$

and since we have assumed $\rho-\rho^{-1} \neq 0,(1)$ is exactly of order $n$ if $\rho^{2 n}=1$.

Let $\gamma \neq 0$. Then equation (2), for $p \neq 2$, has the solution

$$
z=\frac{\alpha-\delta}{2 \gamma} \pm\left(\frac{(\alpha-\delta)^{2}+4 \beta \gamma}{4 \gamma^{2}}\right)^{1 / 2}=\frac{1}{2 \gamma}\left[\alpha-\delta \pm\left(\rho-\rho^{-1}\right)\right]
$$

since $(\alpha-\delta)^{2}+4 \beta \gamma=(\alpha-\delta)^{2}+4 \alpha \delta-4=(\alpha+\delta)^{2}-4=\left(\rho-\rho^{-1}\right)^{2}$, and (1) has the distinct fixpoints $z_{1}, z_{2}$ given by the values of $z$ in (3). Letting $\tau=\left(z-z_{1}\right) /\left(z-z_{2}\right)$ and using $\tau$ as a variable, we find that there is a conjugate of (1) with the fixpoints 0 and $\infty$, and the same trace as (1). Hence it will be of the form $\tau^{\prime}=\rho \tau / \rho^{-1}$, and the lemma is proved for $p \neq 2$.

Let $p=2$. Then $\alpha-\delta=\alpha+\delta, 1+\alpha \delta=\beta \gamma, \rho^{2}+\rho(\alpha+\delta)+1=0$, and $(\gamma z)^{2}+(\alpha+\delta) \gamma z+1+\alpha \delta=0$. Adding, we get

$$
(\gamma z+\rho)^{2}+(\alpha+\delta)(\gamma z+\rho)+\alpha \delta=0
$$

which has as roots the solutions of the linear equations

$$
\gamma z+\rho=\alpha, \quad \gamma z+\rho=\delta .
$$

Now $\alpha \neq \delta$ (or $\alpha+\delta=0$ ), and we have two fixpoints, and can proceed as before. This proves the lemma.

We use the lemma to prove the following.

TheOREM. Let $r, s, t$ be any integers $\geqq 2$. Choose $q$ such that $2 r s t /(q-1)$. (If $r, s, t$ are all odd, we just need $r s t /(q-1)$, and $p$ can be 2.) Then there is a subgroup of $\operatorname{PSL}(2, q)$ generated by two elements $A, B$ such that $A^{r}=B^{s}=(A B)^{t}=1$, and $r, s$ and $t$ are, respectively, the actual orders of $A, B, A B$.

Proof. Let $\alpha, \beta, \gamma$ be elements of the multiplicative group of the Galois field with orders, respectively, of $2 r, 2 s, 2 t$ if $p \neq 2$ and $r, s, t$ if $p=2$. Let

$$
A=\left(\begin{array}{ll}
\alpha & \lambda \\
0 & \alpha^{-1}
\end{array}\right), \quad B=\left(\begin{array}{cc}
\beta+\tau & 1 \\
\mu & \beta^{-1}-\tau
\end{array}\right) .
$$

Then 


$$
A B=\left(\begin{array}{cc}
\alpha(\beta+\tau)+\lambda \mu & \alpha+\lambda\left(\beta^{-1}-\tau\right) \\
\alpha^{-1} \mu & \alpha^{-1}\left(\beta^{-1}-\tau\right)
\end{array}\right) .
$$

If we choose $\lambda, \mu$ and $\tau$ such that

(4) $(\beta+\tau)\left(\beta^{-1}-\tau\right)-\mu=-\tau^{2}+\tau\left(\beta^{-1}-\beta\right)+1-\mu=1=\operatorname{det} B$

and

$$
\alpha(\beta+\tau)+\lambda \mu+\alpha^{-1}\left(\beta^{-1}-\tau\right)=\gamma+\gamma^{-1},
$$

we are finished according to the lemma.

We can satisfy equation (4) with a $\mu \neq 0$ by choosing $\tau$ such that $\tau \neq 0, \tau \neq \beta^{-1}-\beta$. Then we use equation (5) to determine $\lambda$ uniquely, proving the theorem.

$A$ and $B$ then generate a quotient group of $(t, s, r)$ such that there is a homomorphic mapping of $(t, s, r)$ onto it, with the kernel torsionfree.

The author wishes to acknowledge the generous advice of Professor Wilhelm Magnus in the preparation of this paper.

\section{BiBLIOGRAPHY}

1. Wilhelm Magnus, Abraham Karrass and Donald Solitar, Combinatorial group theory: Presentations of groups in terms of generators and relations, Pure and Appl. Math., vol. 13, Interscience, New York, 1966. MR 34 \#7617.

2. Joseph J. Rotman, The theory of groups. An introduction, Allyn and Bacon, Boston, Mass., 1965. MR 34 \#4338.

3. A. Hurwitz, Ueber Riemann'sche Flaechen mit gegebenen Verweigangs punktan, Math. Ann. 39 (1891), 1-61.

4. A. M. Macbeath, Generators of the linear fractional groups, Proc. Sympos. Pure Math., vol. 12, Amer. Math. Soc., Providence, R. I., 1968, pp. 14-32.

5. Ralph H. Fox, On Fenchel's conjecture about F-groups, Mat. Tidsskr. B. 1952, 61-65. MR 14, 843.

6. J. Mennicke, Eine Bemerkung über Fuchssche Gruppen, Invent. Math. 2 (1967), 301-305; ibid. 6 (1968), 106. MR 34 \#7666; MR 38 \#247.

7. J. McCool, Elements of finite order in free product sixth-groups, Glasgow Math. J. 9 (1968), 128-145. MR 38 \#2196.

8. R. Fricke and F. Klein, Vorlesungen ueber die Theorie der automorphen Funktionen, Teubner, Leipzig, 1897, pp. 186-187.

Courant Institute of Mathematical Sciences, New York University, NEW YoRK, NEW YoRk 10012 